\title{
Contra el cansancio y el simulacro: aportes de la filosofía de la educación
}

\section{Against fatigue and simulacrum: contributions from the philosophy of education}

\author{
DOI: $10.46932 / \mathrm{sfjdv3n1-049}$
}

Received in: Dec 30st, 2021

Accepted in: Jan 1th, 2022

\section{Sandra García Pérez}

Licenciada en Pedagogía, Especialista en Docencia, Maestra en Educación Superior y Doctora en Filosofía. Profesora de Tiempo Completo y Tutora Académica y para la Investigación en la Facultad de Pedagogía de la Facultad de Pedagogía en la Universidad Veracruzana. Miembro del Cuerpo Académico Consolidado: "Ciencia, Tecnología, Sociedad e Innovación en la Sociedad del Conocimiento". Reconocimiento a Perfil Deseable del Programa para el Desarrollo Profesional Docente, para el Tipo Superior de la Secretaría de Educación Pública. Línea de Generación y Aplicación del Conocimiento: Innovación social y educativa.

E-mail: sandrigp@yahoo.com.mx

\section{Isis Morales García}

Licenciada en Relaciones Internacionales por la Universidad Nacional Autónoma de México, Licenciada en Administración de Negocios con Mención Honorifica Lux Veritas por la Universidad Anáhuac y Maestra en Innovación y Tecnología por la Universidad IEU. Cursos, talleres y diplomados en Seguridad de la Información, Política de Cumplimiento de la Ley FOREIGN CORRUPT PRACTICES ACT (FCPA) y Actualización de Seguridad de la Información por First Data COMPLIANCE TRAINING \& EDUCATION CENTER. Certificación en Gestión de Mejora KI WO TSUKAU en Organizaciones por el Consejo Nacional de Normalización y Certificación de Competencias Laborales. Distribuidora de NET PAY.

E-mail: isismg@hotmail.com

\section{G. David Suárez Cobix}

Licenciado en Pedagogía con área terminal en Administración Educativa, Maestro en Administración de Personal y Recursos Humanos, así como Doctor en Alta Dirección. Académico del Área de Formación de Elección Libre del Sistema de Enseñanza Abierta en la Universidad Veracruzana. Miembro de la Red Temática CONACyT "Trabajo y condiciones laborales -teTra-" y de la "Sociedad Mexicana de Educación Comparada". Línea de Generación y Aplicación del Conocimiento:

"Personas, estructuras y procesos de las organizaciones educativas".

E-mail: gdcobix@gmail.com

\section{RESUME}

En este escrito, se presenta una reflexión sobre la vigencia de la filosofía de la educación partiendo de diagnósticos que el siglo XX anticipó y que se hacen notar en pleno siglo XXI. El objetivo de este texto es realizar un análisis los fines últimos de un quehacer humano, sobre los humanos. La filosofía de la educación ha sido cuestionada por un horizonte donde aquellos valores e ideales que ella encarnaba, son puestos en duda conforme a la experiencia mundial de cambios y exigencias no consideradas por este saber. Para este ejercicio, nos sustentamos en el análisis documental. Este tema es de suma importancia para el quehacer profesional de cualquier disciplina.

Palabras clave: Filosofía de la educación. Reflexión pedagógica. Fines de la educación. 


\begin{abstract}
In this paper, a reflection is presented on the validity of the philosophy of education based on diagnoses that the twentieth century anticipated and that are noticeable in the twenty-first century. The objective of this text is to make an analysis of the ultimate goals of a human endeavor, about humans. The philosophy of education has been questioned by a horizon where those values and ideals that it embodied, are put in doubt according to the world experience of changes and demands not considered by this knowledge. For this exercise, we rely on documentary analysis. This subject is of utmost importance for the professional work of any discipline.
\end{abstract}

Keywords: Philosophy of education. Pedagogical reflection. Aims of education.

\title{
1 INTRODUCCIÓN
}

El mundo ha cambiado y desde las guerras mundiales hasta la exacerbación de un capitalismo rapaz, que ha creado una era de consumismo y vanidad, incluso en los momentos en que la ciencia y la tecnología pudieron haber solventado problemas sociales radicales, no se ha podido concebir un futuro basado en aquellos ideales que "no resultaron" y dieron pauta al rechazo de la visión clásica y moderna del mundo.

Si bien domina un mundo espiritual y culturalmente fragmentado, sucede que está globalizado por una matriz económica que lo sustenta y que ha permitido una transversalización de actitudes y esquemas de vida que no permiten hacer verdadera comunidad, pues excluyen la unificación de criterios universales relacionados con lo justo, lo bueno, lo razonable, lo humano; todo un sueño imposible, fantástico.

Sólo las grandes emergencias relucen un poco en esta unificación humana mundial fuera de lo económico: primero ha sido la emergencia ecológica, que ha provocado leyes y principios internacionales para atacarla sistemáticamente. Después, la pandemia, que puso en jaque los grandes sistemas de procuración o cuidado humano, como la educación, y a la misma especie humana en general.

Pero en realidad, antes de la pandemia, todo sistema educativo estaba situado en dinámicas donde la preparación profesional y desarrollo humano estaban planteados desde el concepto confuso de competencia. Competencias, habilidades, saberes para un mundo tecno-industrializado y tecnoinformatizado que exige aplicación inmediata y disposición para el trabajo. Desarrollo humano aquí ha significado la preparación en este horizonte para ser competente, que puede significar cosas importantes como la obtención de esos conocimientos y competencias que en verdad son importantes, pero también simbolizan un esquema de existencia en que la vida individualista y jerarquizada es lo primordial.

Todo ello deja de fuera ideales y presupuestos que antaño coordinaron las visiones de lo que es 
el bien y el desarrollo humano. Las ideas de razón, de progreso, de libertad, de justicia, de humanidad son tildadas de elementos metafísicos sin sentido en una era que requiere conocimientos técnicos para la resolución de problemas económicos, reales. Claro que se sigue apelando a elementos tradicionales como el respeto, la tolerancia, la equidad, la bondad, la justicia, la democracia, pero sólo como marco regulatorio para que todo lo demás de realice sin problema.

Las consecuencias de ello en la educación es la disposición de sistemas que no alcanzan a visualizar una configuración realista de los métodos, los contenidos y herramientas para educar a los ciudadanos, ni la manera de resarcir la distancia que está implícita en la asimilación de programas que corresponden a contextos económicos y culturales ajenos. Si las guías para determinar el rumbo de la educación de un país dependen de elementos extranjeros que tienen todas las ventajas para su aplicación, es lógico que se mantenga esta distancia educativa que deriva de las desventajas económicas.

Países en desarrollo no pueden competir con los países desarrollados en la aplicación de programas y modelos educativos que siempre suponen esta ventaja. Por eso, cada modificación que parece poner a los sistemas educativos de estos países en desarrollo en sincronía con lo que ocurre en aquellos que son "ricos", en realidad los sitúa en otro tipo de desventajas que ningún esfuerzo particular puede solventar.

De ahí la decepción de quienes ejercen esta tarea formativa, pues regularmente van a contracorriente tratando de cumplir objetivos y solventar obstáculos que son estructuralmente insuperables. También con un ambiente social, político y cultural que no permite el avance formativo de los estudiantes, pues la violencia y la narcocultura están presentes en su cotidianidad, lo que lleva a normalizarlas. Esta normalización ha provocado un descuido en la atención de la esfera educativa, un desprecio de este ejercicio derivado de la idea generalizada de que todo lo compra el dinero y este se obtienen más rápido y fácil saltando el tiempo que se invierte en la escuela. Es una vinculación tan estrecha entre la vida y el dinero que no permite entender la apertura del mundo, la apreciación de la cultura y de otros modos de vida que puede provocar la escuela y que son importantes para ser persona.

En estos tiempos, en que la pandemia vino a exponer la delicada situación de los caminos educativos y a exacerbar la falta de rendimiento escolar a pesar de y con las muchas herramientas para solventar el problema que representa aún la cuarentena, pensar lo que la educación significa para la humanidad y valorar su estado en cada región es una necesidad, es una forma de tomar las riendas y hacerse responsable de un futuro que estamos dejando como hoja al viendo, en una contemplación que nos hace indiferentes.

Por eso, la reflexión que se presenta quiere participar en este movimiento, incitarlo, haciendo 
énfasis no sólo en la vigencia de la filosofía de la educación y sus elementos primarios, sino en lo que puede ofrecer para el presente una vez que se ha asumido una crisis o un estado cuestionable de la educación, que quienes participan como profesores, como pedagogos, tienen a la mano y viven con una contundencia feroz.

Como se argumenta aquí, el análisis del fenómeno educativo que pedagogos y filósofos de la educación realizan puede alcanzar una mayor profundidad si se combinan y se asume que la educación no puede ser un campo de simulación y que, aún con todo en contra, quienes estamos en este campo no podemos rendirnos y ser pasivos frente a este entuerto. Hay que recordar una de las grandes lecciones de la filosofía de la educación: cuando hablamos de educación, hablamos de la vida humana y sus alcances, de la humanidad en construcción perenne, de comunidad, autonomía y responsabilidad, frente a lo que no cabe la pasividad, la ingenuidad, la indiferencia, la servidumbre, la simulación.

\section{2 ¿QUÉ ES “FILOSOFÍA DE LA EDUCACIÓN”?}

Podemos afirmar, en un primer momento, que la filosofía de la educación es un campo de propuestas de diversos filósofos que desde la antigüedad se han preocupado por pensar y asumir el rumbo o propósitos esenciales del fenómeno educativo. Por lo menos es lo que se puede notar a simple vista cuando revisamos la historia de la filosofía de la educación. En este sentido, estas propuestas están ligadas a la misma historia de la filosofía, pues parten de teorías o sistemas de filósofos aunque representen a diversas traiciones (Vázquez, 2012).

De esto podemos inferir que la filosofía de la educación es filosofía en el sentido más alto, o la presupone (Cohan, 1996: 143). Pero es una filosofía sobre la educación, que denota un pensamiento sobre los fines últimos de un quehacer propiamente humano sobre los humanos. Una proyección general de un estado de humanidad que se debe procurar y a la que los ejercicios educativos deben conducir. Es este sentido, se ha visto a la filosofía de la educación como un simple planteamiento teórico sobre una práctica que lo rebasa o lo supera, por lo que se remarca como un conjunto de utopías que sólo guardan un momento romántico de la especulación. Sin embargo, esto no es así.

Si es propiamente filosofía, podemos entender que la filosofía de la educación sea una reflexión sistemática que ofrece fundamentos sobre la formación de un tipo de persona y un conjunto de valores constitutivo de seres racionales o con capacidades únicas que los hacen inconfundibles con alguna otra especie en el mundo. Se trata de una orientación direccional sobre la humanidad que siempre está supuesta en cualquier idea o teoría educativa. Toda la configuración del acto educativo responde a esta orientación que está implícita o explícitamente en sus objetivos: 
La Filosofía de la Educación es reflexión teórica -formalmente filosófica-sobre el aspecto más formal de la educación: su fin y se halla ordenada sólo mediatamente a la realización de la acción singular. Se trata de un saber teórico-práctico, que fundamenta toda reflexión pedagógica ulterior, la cual puede permanecer como teórico-práctica o ser directamente práctica cuando su fin inmediato es reglar la acción. (Vázquez, 2012: 45)

Por ello, establecer un reconocimiento de la filosofía de la educación no sólo es inevitable, sino trascendente, por lo que implica. Se trata del reconocimiento de las raíces que nutren el fin educativo y que todo aquel que esté involucrado en este acto debe saber y nutrir. Si preguntamos sobre la profundidad de lo que se hace y para qué lo hacemos como profesores, estaremos muy cerca de esta matriz.

José Ma. Quintana es más radical al hablar de este vínculo entre educación y filosofía. Sostiene que la pedagogía es, según lo observado, filosófica:

\begin{abstract}
Pero hay más: la Filosofía es necesariamente pedagógica. La Filosofía, en efecto, está para orientar al hombre; no quiere quedarse en meras especulaciones, sino convertirse en acción. Mas en este último paso la Filosofía deja de ser tal para convertirse en Pedagogía. La Pedagogía no es otra cosa que Filosofía puesta en práctica; por eso, según Dilthey, "la última palabra del filósofo es la Pedagogía, pues todo especular se realiza por el obrar"; "desde un punto de vista general, la floración y fin de toda verdadera Filosofía es la Pedagogía en su más amplio sentido: teoría de la formación del hombre" [8]. La Pedagogía es el coronamiento y consumación de la Filosofía. (Quintana, 1982: 66)
\end{abstract}

Con ello podemos asumir que en toda reflexión educativa hay una tendencia a la filosofía de la educación y que sería muy cuestionable alguna propuesta educativa que no la contenga. Si la pedagogía está tan ligada a la filosofía, no sólo históricamente, sino estructuralmente, entonces no se puede obviar a la filosofía de la educación. Una apuesta por una pedagogía alejada de la filosofía sería así una proyección sin visión, una misión sin rumbo.

Po otra parte, el vínculo de la filosofía de la educación con la teoría de la educación es similar a la que existe entre filosofía política y teoría política. Las teorías son los modos de analizar y nombrar el fenómeno en cuestión sin recurrir a los esquemas, conceptos, principios y métodos universalistas que rechaza de la tradición filosófica. Así lo explica Stella Maris Vázquez:

La teoría, a diferencia de la filosofía, parte de principios que son o bien hipotéticos-en este caso explica, o interpreta una serie de hechos conexos por medio de un modelo-, o bien son el resultado de demostraciones positivo-experimentales. En este último caso la teoría de la educación depende de ciencias positivas, ya sea la biología, la psicología experimental, la sociología. No parte, entonces, de la experiencia inmediata ni procede fenomenológicamente ni busca una reducción a principios evidentes, sino que parte de las conclusiones de aquellas ciencias. (Vázquez, 2012: 47) 
Por eso, la filosofía de la educación es más cercana en objetivos y fines con la pedagogía, sobre todo porque comparten influencias y recorridos históricos que las hacen semejantes y hasta complementarias.

Ahora bien, la filosofía de la educación tiene varias aristas, como sistemas o propuestas filosóficas existen. Algunas son coincidentes en tanto en sus axiomas, como en sus principios y supuestos, pero otras varían de manera importante, por lo que no existe la unificación dentro de este saber. Y esto mismo pasa con la idea de filosofía, donde no hay métodos únicos y sí hay axiomas que se distancian tan radicalmente como aquellos que parten de alguna metafísica y otros que parten de cierto materialismo o positivismo.

A pesar de ello, se habla de filosofía y de sus ramas o especialidades. Pero esto ocurre también cuando se habla de la ciencia, aun cuando no exista un concepto unificado de esta y el método experimental no sea un proceso unívoco, ni uniforme, ni perfecto, ni completamente objetivo, fuera de parámetros que no son propiamente científicos.

Pues bien, siguiendo la idea anterior de que "La Pedagogía no es otra cosa que Filosofía puesta en práctica", como sabemos, la filosofía de la educación se ubica dentro de lo que puede considerarse como filosofía aplicada, que es el esfuerzo de relacionar a la filosofía en su más estricto sentido con una posible praxis en el mundo inmediato; intenta resolver algo y no sólo teorizarlo. No sin problemas, es una clasificación aceptable para nombrar este puente entre la más pura filosofía con asuntos inmediatamente mundanos en los que puede intervenir, como la religión, el derecho o la ciencia.

¿Pero entonces qué es lo que unifica, a pesar de todas estas distinciones, a la filosofía? ¿Qué es, por tanto, lo que unifica a las diversas propuestas de filosofía de la educación? Una respuesta general pero contundente es que la filosofía se define como el esfuerzo racional, por el ejercicio de pensar el mundo y sus diversos problemas; esfuerzo que es crítico y, por tanto, tiene una tendencia reformadora. Eso que nombramos "sentido" es uno de los elementos que está implícito en esta búsqueda, aquello que tiene sentido es lo que refleja nuestra racionalidad y nuestra humanidad, algo que podemos y debemos hacer para alcanzar o perpetuar un bien humano.

Según esto, la filosofía de la educación es el esfuerzo racional de pensar y (tiene el interés de) resolver los asuntos involucrados con la educación en general (Aguilar, 2020: 100), desde el propio concepto hasta sus fines, métodos y fundamentos. Además de todo lo que históricamente se va derivando como problemática educativa o pedagógica. Y es que, la realidad educativa debe cuestionarse constantemente, con

[...] un análisis, una valoración crítica constante y una reflexión sistemática; tareas estas últimas que puede llevar a cabo la filosofía de la educación si por ella entendemos una rama 
de la filosofía, distinta a las ramas tradicionales de aquélla pero no independiente ni mucho menos autosuficiente, que discute la educación y educabilidad humanas. (Martínez, 2015: 59)

Por otro lado, si la Pedagogía tiene estos alcances como reflexión y praxis (Vázquez, 2012: 4041), entonces se comprenderá que pedagogía y filosofía de la educación entonces sí tienen esta cercanía que mencionaba José Ma. Quintana.

Algunos de estos asuntos son: los fines de la educación, la perfectibilidad y libertad humanas, la concepción del mundo y de la vida, los valores, la ética, el derecho y deber de educar, los sistemas pedagógicos, la visión del hombre, los procesos pedagógicos y sus implicaciones filosóficas, los fundamentos educativos (Quintana, 1982: 70). La conclusión de Quintana sobre lo que es la filosofía de la educación es la siguiente:

"Filosofía de la Educación es la explicación filosófica de la educación, y la elaboración crítica de los principios ideológicos que presupone el acto educativo y que sirven para orientarlo" (la primera parte de la definición alude a una Filosofía de la Educación "especial", y el resto a una Filosofía "aplicada"). (Quintana, 1982: 72)

Podemos agregar, sin duda, otros elementos que acompañan a la idea general de filosofía de la educación, además de la racionalidad y la crítica, que serían los fundamentos para justificar la capacidad reformadora que tiene y, por tanto, la importancia de su ejercicio concentrado en el análisis y práctica de la educación.

Ya sea como orientación o saber racional y crítico de las posibilidades de la realidad educativa en su conjunto (Fullat, 2000: 12), como teoría y praxis, la filosofía de la educación lleva en sí las simientes de una reformulación de todo lo analizado del fenómeno educativo.

\section{LA FILOSOFÍA DE LA EDUCACIÓN COMO AUTOCOMPRENSIÓN Y AUTOCRÍTICA}

La filosofía conlleva, desde sus inicios, no sólo la actitud contemplativa, que ha generado esta visión universal de la filosofía como teoría pura o conjunto de especulaciones fuera del rango de lo practicable, es decir, con esa idea generalizada de la metafísica como "más allá de lo físico". Lo que se obvia en esta generalización es que desde sus inicios, la filosofía no sólo es altamente especulativa, sino crítica.

Fueron los griegos quienes cultivaron especialmente este pensamiento que desembocó en lo que conocemos como filosofía occidental, configurada en un espacio político-cultural particular que permitió esto que podemos nombrar actitud filosófica: la atención a lo asombroso de la existencia en general y la mordacidad analítica que ponía en crisis los elementos no cuestionados de la vida cotidiana y los actos normalizados. 
La actitud contraria sería lo que Husserl ha llamado "actitud natural" (Rabanaque, 2011) que, a grandes rasgos, expresa la insistencia de un giro de un conjunto de acciones acríticas, naturalizadas, que evitan la tarea de pensar. No pensar sería asumir las condiciones externas a las que toda acción respondería sin ninguna mediación. Dejarse lleva, asumir lo que la mayoría dice o lo que "se dice" o "se hace", encarnar la idea de alguien más sin haberla pasado por la criba de la razón, son otras maneras de nombrar esta actitud.

Si bien la definición de "filosofía" es un problema interno como el de muchos otros saberes, podemos situar su unidad en la actitud primaria que evoca su puesta en marcha: una actitud contemplativa y crítica. Así, cuando hablamos de filosofía de la educación, estamos suponiendo esta base unificadora de esfuerzo racional que apunta con especial atención y actitud crítica hacia lo que constituye la educación. Por más que los estudios contemporáneos estén elevando los procesos cognitivos a esquemas igualitarios entre diversas especies, es el hombre y sus capacidades de pensar en general las que sustentan y siguen dando sentido al mundo, en su realidad crítica y en su emergencia de salvarlo (Ramírez, 2015: 175-179).

Por lo anterior, si se habla de la filosofía de la educación, podemos pensar tanto en esta variedad de propuestas sobre la génesis y teleología de la educación, como de uno de los actos que parecen más modestos en el hombre: la reflexión sobre sí y su quehacer, que implican este movimiento de contemplación, asombro y crítica. En el caso de quien ha asumido a tarea de educador, esta atención a su quehacer que no cesa conlleva el carácter primordial por el que tiene sentido situarnos en la filosofía de la educación:

En efecto, es preciso desarrollar un proceso de reflexión sobre los problemas educacionales de nuestro tiempo. Ese proceso no puede no desarrollarse desde dentro de la filosofía y la educación. La filosofía no debe ser considerada una cosa acabada y externa sino una actitud metódica del profesor-educador que enfrenta reflexiva y críticamente esa realidad educacional de la que forma parte. (Cohan, 1996: 143)

Como bien sostiene Walter Cohan al hablar de la enseñanza de la filosofía, cuando hablamos de esta acción de enseña filosofía, según Kant, no es posible mostrarla como algo acabado y sin problemas, mucho menos como algo ajeno a quien la enseña. De alguna manera, enseñar filosofía implica una praxis de ella por parte de quien la lleva en la voz, de alguna manera se vive y se sume alguna de sus múltiples enseñanzas para la vida.

De ahí la profundidad de la aclaración kantiana de que aprende a filosofar. Para él, precisamente, y atendiendo al ejemplo socrático, filosofía es una habilidad (una capacidad), por eso no se transmite, sino que se provoca, porque en su esencia, es una acción llevada a cabo por la facultad más alta del hombre; pero también quiere decir utilidad, ya que lleva a quien la practica a la sabiduría, 
a un alejamiento de la pura doxa (opinión). En este sentido, la filosofía para Kant es la "legisladora de la razón" (Kant, 2000: 91). Si asumimos aún que el elemento más propio del hombre es su capacidad de razonar, entonces tendremos que asumir la necesidad de filosofar.

Según Cohan, así como la filosofía no es vivencialmente ajena a quien "la enseña" y la tarea de esta enseñanza no es sino filosofar: "Para la filosofía de la educación vale lo mismo que para cualquier otra 'filosofía de': enseñarla comporta hacerla, practicarla, vivirla. Esto implica un movimiento reflexivo, problematizador de la realidad educativa contemporánea" (Cohan, 1996: 144).

Este énfasis en la reflexión sobre la realidad educativa contemporánea es remarcado por el autor para reconocer la historicidad de la filosofía y de la filosofía de la educación. Como hemos dicho, se trata de la dimensión temporal o contingente en que todo fenómeno humano se da, de tal manera que cada problema contemporáneo no se resuelve con respuestas del pasado. La contemporaneidad tiene sus propios rasgos y exige respuestas apegadas a ellos, no repeticiones. Por tanto, "no reconocer esa dimensión empobrece la búsqueda, limita los caminos a recorrer, reduce las alternativas, estrecha, al fin, las posibilidades de pensar los problemas actuales de la educación (Cohan, 1996: 146).

Pero también es importante mencionar que una tarea constante, que forma parte del despliegue de todo filosofar, es analizar, revisar, evaluar todo lo que se está constituyendo como presente: hacia dónde se dirige, por qué y cómo lo hace. Especialmente, la filosofía de la educación tendría que enmarcarse en el cuestionamiento constante del quehacer educativo y cuestionar qué tipo de sujeto está apuntalando cada sistema educativo, tener en claro que cada acción llevada a cabo coadyuva a la configuración de sujetos con especiales características o "competencias":

Así, la filosofía de la educación procura comprender aquello que la educación, en su particularidad frente a otras prácticas sociales, constituye en una de sus funciones más salientes: la conformación de determinado tipo de sujetos. Esta comprensión comporta el análisis riguroso y pormenorizado de las ideas, valores y creencias que subyacen y se siguen de una práctica educacional. (Cohan, 1996: 150)

Esta sería una tarea primordial para una filosofía de la educación: comprender la trama humana tal y como se desarrolla en el presente y hacer una crítica de los modos en que se constituye un ejercicio de formación y desarrollo humano, haciendo énfasis en las ideas, valores y creencias presentes, expuestas o no en práctica educacional, considerando que son los elementos que forman subjetividades y las vinculan de cierta manera con el mundo. La aportación entonces se dirige a que la educación cumpla una de sus principales tareas:

[...] el desarrollo de una autonomía, de un sujeto autónomo que implica [...] el desarrollo de esas capacidades que nos definen, y que cuentan necesariamente con los otros. En la jerarquía de esas capacidades quizás la que orienta todas, la capacidad suprema -y lo que conlleva decir, por tanto, el máximo fin de la educación-, es la capacidad de responsabilidad; el sujeto 
responsable supone y presupone un sujeto capaz de decirse, de actuar, de narrar, etc. (Ramírez, 2015: 152)

\section{LA FILOSOFÍA DE LA EDUCACIÓN COMO RESISTENCIA, LIBERACIÓN Y RENOVACIÓN}

Uno de los resultados inmediatos de este ejercicio implícito en la filosofía de la educación es la posible ruptura de las condiciones negativas o irracionales que se puedan detectar, sobre todo en los modos en que se pueden presentar condiciones anti-pedagógicas o negativas para los objetivos de la educación.

La filosofía de la educación, como toda filosofía, es un compromiso con la racionalidad del hombre, la verdad, la justicia y todos aquellos bienes a los que se puede comprometer el ejercicio de la razón. Eso es lo razonable, aunque suene a ideales completamente utópicos y "metafísicos" (en el significado negativo que se le ha querido establecer a la metafísica desde hace mucho tiempo).

Así, lo razonable en la educación es aquello que se apega a los más altos ideales humanos y que ningún interés inmediato, individual, egoísta, sombrío puede manipular. Es la libertad y honestidad de quien se hace responsable de una terea que es siempre histórica y siempre perfectible, pero irrenunciable; búsqueda de la libertad situada (Vázquez, 2012: 33). Quizá eso es lo que muchos quieran decir cuando afirman que tienen vocación, que conlleva su rasgo ético.

Por lo tanto, la filosofía de la educación puede considerarse un alto en el camino del trabajo educativo para sopesar lo caminado y sacudir aquellos obstáculos que se van formando y naturalizando como si ya no hubiera más opciones. Son los obstáculos del tiempo que generan una miopía y actitud ingenua que confundimos con el destino, con lo inevitable, como si se arrinconara a la libertad.

Por eso es importante destacar el aspecto crítico de la filosofía en general y de la filosofía de la educación: "Esta función propuesta para la filosofía de la educación permite avizorar la fragilidad del presente orden de las cosas y, al mismo tiempo, abre las puertas para pensar de otras maneras aquello que hoy es pensado como inmodificable" (Cohan, 1996: 151).

Esta atención hacia un recorrido es el modo de autorreflexión que permite evaluar y sopesar hasta dónde lo que hacemos está bien y genera beneficios para el desarrollo humano (autocomprensión). La pregunta que surge es: ¿cómo el que hace este ejercicio se puede liberar de las condiciones que han estado presentes en su quehacer incluso antes de haber nacido? ¿Desde qué horizonte se puede parar, tomar distancia, analizar y emprender una crítica que puede desmoronar todo?

La respuesta está en la posición ilustrada, más vieja que la Ilustración francesa, que sigue guiando a las propuestas más novedosas sobre metodología educativa: que todo hombre tiene la facultad de pensar y actuar por sí mismo, considerando siempre lo mejor para sus congéneres. La 
facultad de pensar, que en varios autores se despliega como la facultad de dudar o de preguntar, es la posibilidad de la autonomía del sujeto, eso que pretenden las propuestas más novedosas en educación. Autonomía, autocomprensión, autocrítica, autorrealización serían las maneras de decir esta especie de naturaleza humana que, por cierto, siempre ha incluido la moderación de un pathos, también natural.

Sólo realizando estas posibilidades humanas es que se puede hacer una crítica radical a los estados en que la política, la cultura, la sociedad y los sistemas educativos han caído (autocrítica). Sólo este hacer pensante puede provocar renovaciones y anticipar tropiezos, pensar utopías y superar distopías. Se trata de una de las grandes metas de la educación: crear agentes libres, libre-pensadores que pueden resolver los problemas viejos y nuevos del mundo, hacer de él un mundo mejor:

\footnotetext{
El hombre es un ente que nunca ha cumplido del todo. Hállase en constante tensión de cumplimiento hasta alcanzar la muerte. Si el hombre es "deber-ser" se debe a que antes es "poder-ser", es perfectibilidad, es libertad. Cuando el educador se vierte a su tarea no puede, por tanto, perderse en proporcionar un acopio instructivo de noticias al educando; tiene que despertarle el "deber-ser", la libertad. (Fullat, 2000: 106)
}

Fullat parece seguir aquí a Kant, quien afirma: "El verdadero filósofo, como libre pensador, debe usar propia e independientemente de su razón y no emplearla de una manera servil" (Kant, 2000:93). Esta es la relación esencial entre filosofía y filosofía de la educación: la búsqueda de la libertad y la realización humanas y el rechazo de estados serviles (estados de infancia, decía Kant) donde las capacidades y libertad humanas son sustituidas por la obediencia, la esclavitud, lo inhumano.

Así, en tanto que la filosofía de la educación se plantea como un ejercicio de resistencia y crítica de esos estados negativos en los que puede caer conscientemente o no el hombre, también se plantea como uno de los modos de emprender etapas de renovación humana con el objetivo de mantener el espacio de libertad y desarrollo humano que siempre supone el acto educativo. La filosofía de la educación es un modo de comprender que el hombre es un fin en sí mismo y no un medio.

\section{CONCLUSIONES.}

1. La filosofía de la educación no es un saber acabado que indique un cúmulo de propuestas incuestionables. Si bien está apegada a su historia, como la filosofía misma lo está, la apreciación de ella debe darse a partir de los problemas reales y contemporáneos de la educación. De ahí que todo verdadero punto de partida sea, como nos dice Ortega y Gasset, la circunstancia. Con ello se logrará recobrar el sentido y mensajes de la historia de la filosofía de la educación y hacerlo un saber siempre situado.

2. En tanto saber complejo, como todo saber, la filosofía de la educación es difícil de definir. Hay varias aristas desde dónde hablar de ella: desde la epistemología, desde la teoría, desde su 
enseñanza, desde su práctica, desde su puesta en marcha como teoría, desde su historia. Pero uno de las cosas esenciales es su relación con la misma filosofía, que la dota de un carácter primordial y más unívoco (aunque al situarla, siguiendo Beuchot, estemos forzando su dimensión analógica). Por este vínculo podemos expresar ciertas tareas y compromisos a los que la filosofía de la educación no puede renunciar y que permiten comprender la responsabilidad del fenómeno educativo y la necesidad de pensarlo.

3. Por su raíz filosófica, la filosofía de la educación es el esfuerzo de pensar los asuntos involucrados con la educación: el propio concepto, sus fines, métodos y fundamentos. En este sentido, en las propuestas de la pedagogía siempre está involucrada la filosofía de a educación como fondo de una visión ejecutable. Si los pedagogos, los profesores, los teóricos o investigadores de la educación pueden hacer un análisis de los problemas educativos o pedagógicos, la filosofía de la educación les proporciona un marco histórico, conceptual y argumental con el que pueden hacer su crítica y respectiva propuesta. La realidad educativa es cuestionable y siempre va a exigir esta atención histórica, conceptual, racional que la filosofía de la educación proporciona.

4. Precisamente, una tarea primordial de la filosofía de la educación es comprender la trama humana tal y como se desarrolla en el presente educativo e influir en él. Se trata de detenerse, analizar, comprender las ideas, valores y creencias presentes en práctica educacional, en tanto elementos que forman subjetividades y las vinculan de cierta manera con el mundo. De ahí que la filosofía de la educación pueda ser siempre una posibilidad de crítica a los ejercicios de formación y desarrollo humano.

La visión que la guía es el desarrollo de la autonomía humana, de las capacidades supuestas en el acto educativo que nos definen como individuos humanos. Entre ellas, la racionalidad que implica la búsqueda de una vida libre, responsable, comunitaria, realmente humana.

5. Así, la filosofía de la educación implica un compromiso con la racionalidad del hombre, la verdad, la justicia y todos aquellos bienes a los que se puede comprometer el ejercicio de la razón. Lo razonable es el desarrollo y perpetuidad de las capacidades humanas, todo aquello que puede hacerlo vivir plenamente, en la dimensión intersubjetiva que compone a la individualidad.

Lo contrario, lo irracional, que puede ser detectado por este ejercicio filosófico, tendría que asumirse como un obstáculo para la libertad y la responsabilidad. Por ello aquí se ha destacado el aspecto crítico de la filosofía en general y de la filosofía de la educación. La crítica es la posibilidad de alejarnos de los estados serviles. Si no hay crítica, se presenta el riesgo del dominio de la obediencia, la esclavitud, lo inhumano, el cansancio, la simulación.

Por tanto, en su esencia, la filosofía de la educación es un ejercicio de resistencia y destrucción de 
estados negativos en los que puede caer el hombre, así como la manera de emprender la renovación humana con el objetivo de mantener el espacio de libertad y desarrollo que siempre supone el acto educativo. 


\section{REFERENCIAS}

AGUILAR, F. R. (2020). Contribuciones de la filosofía para la consolidación de la filosofía de la educación. Revista Conrado, 16(74), 99-111.

COHAN, W. (1996). Filosofía de la educación: algunas perspectivas actuales. Aula 8, Universidad de Salamanca (141-151).

FULLAT, O. (2000). Filosofía de la educación. España. Síntesis.

KANT, I. (2000). Lógica. Un manual de lecciones. Madrid. Akal.

MARTÍNEZ, (2015). La Filosofía de la Educación de Paulo Freire. Revista Internacional de Educación para la Justicia Social (RIEJS), 4(1), 55-70. doi: http://dx.doi.org/10.15366/riejs2015.4.1

QUINTANA Cabañas, J. Ma . (1982). Concepto de Filosofía de la Educación. Revista Española de Pedagogía. Año XL, No. 157 (Junio-Septiembre).

RABANAQUE, L. R. (2011). Actitud natural y actitud fenomenológica. Sapientia, 67.229-230. Disponible en: http://bibliotecadigital.uca.edu.ar/repositorio/revistas/actitud-natural-actitudfenomenologica-rabanaque.pdf

RAMÍREZ, I. (2015). Voces de la educación. México. Ediciones del Lirio.

VÁZQUEZ, S. (2012). La filosofía de la educación: estado de la cuestión y líneas esenciales. CIAFIC Ediciones. 